\title{
Retrospectiva histórica e temáticas investigadas nas pesquisas empíricas sobre o processo de preparação da performance musical
}

\author{
Luís Cláudio Barros (UDESC, Florianópolis, SC) \\ luisclaudiobarros@yahoo.com.br
}

Resumo: A partir de uma retrospectiva histórica e de contextualização da pesquisa empírica sobre o planejamento da execução instrumental, o presente trabalho estabelece o panorama das principais vertentes temáticas examinadas pela pesquisa científica. Ciente de que o objeto de estudo possui muitas ramificações, abordagens e especificidades, grande parte dos temas de pesquisa pode ser enquadrada dentro de algumas categorias temáticas de maior abrangência. Nesse contexto, o artigo define as categorias em que cada tema relacionado às etapas de preparação da performance musical pode ser inserido no campo da pesquisa empírica em Práticas Interpretativas.

Palavras-Chave: Pesquisa em música; Temáticas em Práticas Interpretativas; preparação da execução instrumental.

\section{A historical retrospective and research subjects of the empirical research in performance planning}

Abstract: This article gives an overview of the main subjects investigated by the scientific research from an historical retrospective of empirical research in Performance Planning. Knowing that the research object has many ramifications and specificities, it is possible to insert a great part of the subjects of research within a broad thematic category. In this context, this article defines the categories in which each theme related to performance planning can be inserted within Musical Performance.

Keywords: Music research; Subjects in performance; Musical Performance Planning.

\section{1 - Aproximação ao Problema}

O presente artigo realiza uma retrospectiva histórica das pesquisas empíricas que examinaram o processo de preparação da performance musical, englobando temáticas que vão desde a leitura à primeira vista da obra musical até a sua performance final. Essas etapas de organização do estudo do instrumento e aprendizagem de um repertório musical são chamadas por GABRIELSSON (2003, p.223) de "Planejamento da Execução Instrumental", sendo inseridas em uma das subdivisões de pesquisa em Práticas Interpretativas.

Após apresentar a contextualização histórica desta linha de pesquisa, o artigo discorre sobre as vertentes temáticas dos trabalhos empíricos realizados entre 1980 e 2008 sobre o planejamento da execução instrumental. A opção por esse período justifica-se por ser a época em que se delimitam e se concentram, em número crescente de publicações, as pesquisas sobre a temática.

Foi elaborado um plano organizacional que categorizou os inúmeros artigos coletados, estabelecendo-se critérios de seleção e de ordenamento dos trabalhos de 
acordo com o método de pesquisa empregado: pesquisas experimentais, pesquisas descritivas com delineamento experimental, estudos de caso, estudos com entrevistas, questionários, levantamentos/survey, dentre outros. Nesse contexto, delimitou-se o espectro investigativo das pesquisas empíricas sobre as estratégias, as técnicas e métodos empregados na prática do instrumento, a organização do estudo e as etapas do processo de aprendizagem.

Em relação ao objeto observado, qual seja, o músico e sua prática instrumental, as pesquisas empíricas estão centradas em dois eixos sobre 0 desempenho instrumental: (1) o músico profissional com nível de expertise, atuando como modelo da ação músico-instrumental e servindo de parâmetro para o ensino do instrumento (CHAFFIN et al., 2002, 2005; MIKLASZEWSKI, 1989; ERICSSON e RAMPE (1996) e (2) os estudantes de música e músicos amadores, em seus variados estágios de aprendizagem e níveis de habilidade musical, os quais são objetos de estudo para exame dos aspectos específicos da execução musical, como o tipo de prática empregada, as diferenças individuais durante o estudo, a estrutura da prática, 0 efeito das estratégias de estudo, os aspectos motores ou expressivos da execução e a memorização (BARRY, 1992; BURNSED e HUMPHRIES, 1998; COFFMAN, 1990; COSTA, 1999; DAVIDSON e MCPHERSON, 2000; ERICSSON et al. 1993; GRUSON, 1988; HALLAM, 2001; HALSBAND et al. 1994; MISHRA 2002; ROSENTHAL et al. 1988; ROSTRON e BOTTRILL, 2000; WILLIAMON E THOMPSON, 2003; WILLIAMON e VALENTINE, 2000, 2002; WOODY, 2003; dentre outros). Esses dois eixos sobre o desempenho instrumental desencadeiam as investigações sobre as referidas temáticas.

\section{2 - Definições de Planejamento da Execução Instrumental}

Este artigo restringe-se à linha de pesquisa ${ }^{1} \mathrm{n}^{-} 5$, chamada por GABRIELSSON (2003) de Planejamento da Execução Instrumental. No mapeamento de GABRIELSSON, essa vertente investigativa é representada por trabalhos escritos entre 1995 e 2002, destacando os seguintes autores: CHAFFIN et al. $(2001,2002)$; CLARKE (1988); DRAKE et al. (2000); ERICSSON et al. (1993, 1997, 1998); GINSBORG (2002); GRUSON (1988); HALLAM (1995, 1997); KRAMPE (1997); LEHMANN (1997, 1996, 1998); MIKLASZEWSKI (1989, 1995); MACPHERSON e MCCORMICK (1999); NIELSEN (1997, 1999, 2001); O’NEILL (1997, 1999, 2002); SLOBODA (1996); SULLIVAN e CANTWELL (1999); PALMER e MEYER (2000); PALMER e VAN DE SANDE (1995); WILLIAMON e VALENTINE $(2000,2002)$. Entretanto, GABRIELSSON não realizou uma análise crítica do conhecimento produzido sobre o planejamento da execução, visto que seu foco foi fornecer principais fontes bibliográficas e a discriminação das dez linhas de pesquisa em Práticas Interpretativas. Outros trabalhos que trouxeram contribuição para a linha de pesquisa $\mathrm{n}^{\circ} 5$ foram examinados e acrescidos ao presente artigo.

A conceituação de planejamento da execução foi indispensável para realizar uma análise precisa e imparcial, provendo foco e prevenindo eventuais desvios do cerne investigativo. As definições foram retiradas dos referenciais teóricos do trabalho e 
serviram para delimitar as pesquisas e as fontes bibliográficas percorridas e os limites temáticos pertencentes à linha de pesquisa. Assim, entende-se por planejamento da execução instrumental:

2.1. A sistematização e organização consciente e refletida da prática diária do instrumento através de um conjunto de estratégias e técnicas de estudo (utilizadas com um objetivo específico a ser alcançado) as quais irão, indubitavelmente, otimizar os resultados da ação músico-instrumental. Essas características estão relacionadas ao que HALLAM (1997c) denomina de metacognição. Para essa autora, as estratégias metacognitivas estão centradas no planejamento, monitoramento e avaliação do aprendizado (p.209). Segundo CHAFFIN (WILLIAMON, 2004, p.28), o músico deve ter uma grande variedade de estratégias para serem utilizadas flexivelmente, de acordo com a finalidade do estudo e as dificuldades a serem solucionadas. HALLAM ressalta que músicos profissionais detêm o controle sobre a prática instrumental, pois esses têm a "necessidade de ajustar continuamente os processos de planejamento, coletando informações, formulando hipóteses, fazendo escolhas e reconsiderando decisões", ou seja, "alcançando o produto-alvo no menor tempo possível" (1997c, p.181). Essas características são atributos do que considera como uma prática efetiva, tendo como modelo o exame sobre a prática de estudo do músico profissional. Sua pesquisa descritiva de $2001 \mathrm{~b}$ sugere que o aumento no nível de planejamento da prática instrumental pode ser uma característica necessária para se tornar um músico de excelência (p.13);

2.2. $\quad$ estudo do instrumento baseado em resultados qualitativos acima dos quantitativos, partindo-se do pressuposto de que o conteúdo e a qualidade da prática são mais importantes do que a quantidade de horas despendidas na prática (WILLIAMON e VALENTINE, 2000). A qualidade subentende um constante monitoramento pela própria pessoa do seu desempenho técnico-motor em relação à produção sonora. Nesse sentido, REID (2002, p.110) postula que a prática deve ser vista como uma atividade que busca solucionar problemas (problem-solving activity), em que o músico identifica a dificuldade para encontrar meios de eliminá-la. Ressalto que essas soluções devem ser estabelecidas a partir da resultante sonora, estabelecendo uma conexão onde a qualidade do estudo e da execução relaciona-se à qualidade da sonoridade produzida;

2.3. A maneira como a representação mental e a estrutura da música influencia a execução e a organização da prática instrumental (CHAFFIN et al., 2002). Para WILLIAMON (2004, p.28), as decisões iniciais sobre a técnica devem ser embasadas sobre a ideia musical e os objetivos expressivos finais da execução. Sem isso em mente, muitas dessas decisões poderão ser mudadas futuramente, ocasionando um prolongamento do período de aprendizagem. A representação mental é fundamentada nas experiências musicais prévias, ou seja, na familiaridade que temos com a obra em 
questão, estabelecidas pela apreensão, entendimento e concepção imaginária da peça musical;

2.4. As etapas e o conteúdo do processo de aprendizagem que levam a uma execução em nível de expertise. Segundo WILLIAMON (2004, p.8), o refinamento da habilidade musical é proveniente de uma prática de estudo altamente eficiente após anos de experiência. Outros autores também destacam que "o nível de proficiência em um determinado domínio de conhecimento é uma função direta da quantidade e qualidade de esforço na prática estruturada das habilidades específicas que o compõem" (ERICSSON et al.; SLOBODA citado por BORÉM et al., 2002, p.18). De acordo com ERICSSON et al. (1993, p.14), um padrão de desempenho superior vai além do domínio do conhecimento e da habilidade com o instrumento, visto requerer contribuições inovadoras e relevantes para a área. Assim, um músico eminente deverá fornecer novas ideias, teorias, métodos, técnicas e interpretações distintas da música que executa.

\section{3 - Retrospectiva histórica das pesquisas empíricas sobre 0 Planejamento da Execução Instrumental}

JORGENSEN (WILLIAMON, 2004, p.87) apresenta uma retrospectiva histórica das pesquisas empíricas sobre o Planejamento da Execução Instrumental. Segundo fontes bibliográficas, a data de 1916 marca a publicação do primeiro trabalho sobre os aspectos da preparação de um repertório a partir de um procedimento prático realizado pelo pianista húngaro Sandor Kovacs. Esta pesquisa investigou meios de aprimorar a memorização e, em especial, a importância do estudo mental da obra no início do processo de aprendizado de um novo repertório. Os resultados mostraram que a retenção dos fragmentos musicais foi superior quando aprendidas longe do instrumento (HALLAM, 1997, p.207). Nas duas décadas subseqüentes a esse estudo, apenas três trabalhos foram publicados: BROWN $(1928,1933)$ examinando a relação entre segmentação e não-segmentação da obra musical durante a prática e EBERLY (1921) investigando a coordenação entre as mãos durante a execução. O trabalho de BROWN (1928) é considerado um dos primeiros estudos experimentais sobre estratégias de estudo. Alguns anos depois, entre 1937 a 1947, a psicóloga e educadora americana Grace Rubin-Rabson publicou uma série de dez estudos sobre o comportamento durante a prática do instrumento. WILLIAMON E VALENTINE (2000, p.356) criticam muitos dos trabalhos de Brown e Rubin-Rabson por terem investigado a prática do instrumento e a memorização requerendo apenas que os sujeitos aprendessem pequenas seções musicais. Assim, muitas das conclusões extraídas desses estudos tendem a ser limitadas, visto que esses trechos musicais curtos não representam a complexidade e o esforço total requerido no aprendizado de um repertório de concerto. Conseqüentemente, não proporcionaram uma situação de aprendizagem "real e familiar" ao músico para que o fenômeno fosse examinado em contexto. Apesar dessas críticas, os trabalhos sobre memorização do repertório pianístico de RUBIN-RABSON (1937, 1939, 1940a, 1940b, 1941) são sempre citados por pesquisadores que investigam os aspectos relativos à memorização e por terem sido estudos pioneiros sobre as estratégias da prática instrumental envolvidas no 
processo cognitivo de assimilação mental do repertório. Conforme COFFMAN (1990, p.189), Rubin-Rabson foi a primeira pesquisadora a examinar a prática mental e a mostrar que o estudo mental da partitura é superior à prática física (de execução) para reter o conteúdo musical na memória.

O’BRIEN (1943), autor da área da psicologia, averiguou algumas estratégias específicas utilizadas durante o estudo instrumental, como a organização da prática a partir de trechos musicais curtos e longos e sua influência na memorização. Outro pioneiro na área da psicologia da música é C. E. SEASHORE (1937, 1938/1967, 1939), sendo amplamente citado por autores de pesquisas sobre a prática deliberada, principalmente o trabalho publicado em 1939, abordando a extensão do tempo gasto na prática. Alguns outros experimentos sobre aspectos interpretativos da execução musical, como altura, timbre, tempo e intensidade foram descritos no livro publicado em 1938 (MIKLASZEWSKI, 1989, p.95).

WICINSKI (1950) e MANTURZEWSKA (1969) também desenvolveram trabalhos precursores coletando dados sobre músicos profissionais. WICINSKI realizou um estudo de entrevistas com pianistas russos eminentes, dentre os quais, Richter, Gilels e Neuhaus. Objetivou-se averiguar como esses músicos estudavam uma nova peça musical. A partir da prática do instrumento, os músicos foram categorizados em dois grupos: (1) o primeiro formado pelos pianistas que dividiam o aprendizado em três etapas (o conhecimento da música e a formação das ideias preliminares sobre como a peça deveria ser executada; o trabalho intensivo nos problemas técnicos; a fusão das duas primeiras etapas com o objetivo de formar a versão interpretativa final) e; (2) o segundo grupo, constituído por pianistas que tinham uma visão global e orgânica do processo de aprendizagem, os quais não dividiam o estudo em etapas, mas o organizavam de acordo com a situação. MANTURZEWSKA (1969) efetuou um estudo através de entrevistas com participantes do Concurso Internacional de Piano Frédéric Chopin para verificar como esses estruturavam sua prática instrumental. A autora relatou que houve significativas diferenças entre os músicos. Todos os pianistas estudavam muitas horas e com estratégias de estudo semelhantes. Os premiados, porém, tinham uma prática muito mais sistemática, regular e disciplinada, preferindo estudar a realizar outras atividades (WICINSKI, 1950; MANTURZEWSKA, 1969, citada por MIKLASZEWSKI, 1989, p. 96).

A partir de 1980, houve uma intensificação nas pesquisas sobre planejamento da execução e após 1990 houve um aumento considerável do número de trabalhos publicados. Segundo GABRIELSSON (2003, p.236), as pesquisas sobre 0 planejamento da execução instrumental iniciaram-se tardiamente na área de Práticas Interpretativas, ou seja, somente a partir da segunda metade do século passado com a emergência da psicologia cognitiva, através do entendimento de como se formam as representações mentais da música e as estratégias para uma prática eficiente. As razões para a intensificação dos trabalhos a partir do final da década de 1980 foi devido aos novos recursos tecnológicos oferecidos, como o registro em vídeo e áudio e, posteriormente, a utilização de programas de computador para a análise dos dados coletados, além do crescente interesse dos pesquisadores na temática. JORGENSEN (WILLIAMON, 2004, p.87) relata que alguns resultados conflitantes 
têm aparecido no corpo dos estudos empíricos desde 1916. Todavia, não evidencia quais seriam as inconsistências encontradas. Uma das razões para a falta de legitimação ou abertura para questionamentos em algumas dessas investigações talvez esteja centrada na fragilidade do referencial teórico utilizado.

Um fator que vincula as pesquisas sobre o planejamento da execução à área de psicologia da música é o fato de que um maior desenvolvimento dessas pesquisas coincidiu também com o período em que houve um incremento dos trabalhos em psicologia da música. Segundo WILLIAMON e THOMPSON (2004), foi somente a partir dos últimos trinta anos que a psicologia da música emergiu como um campo unificado de pesquisa com o surgimento de Sociedades Internacionais (como, por exemplo, o European Society for the Cognitive Sciences of Music e International Society for Music Perception and Cognition) e o lançamento de importantes periódicos da área de psicologia da música: Psychology of Music, em 1973, Psychomusicology, em 1982, Music Perception, em 1983 e Musicae Scientiae, em 1997.

\section{4 - Temáticas abordadas pela linha de pesquisa do Planejamento da Execução Instrumental}

A retrospectiva histórica se ateve aos estudos empíricos pioneiros, fornecendo um breve panorama sobre o que foi realizado na referida linha de pesquisa antes de 1980. Contudo, para a categorização das temáticas investigadas pelo Planejamento da Execução, o presente artigo se concentrou nos trabalhos publicados a partir de 1980, por se tratar de pesquisas mais recentes e metodologicamente bem construídas.

As temáticas investigadas podem ter muitas ramificações, abordagens e especificidades, sendo que grande parte dos temas de pesquisa pode ser enquadrada dentro de três categorias de maior abrangência:

4.1. Temáticas relativas à análise do comportamento durante o estudo; organização, características e tipos de prática;

4.2. Temáticas que abordam as estratégias de estudo;

4.3. Temáticas que abordam a representação mental da música e processos cognitivos envolvidos na memorização.

A subdivisão em três categorias visa à organização das referências disponíveis, ou seja, tem uma finalidade didática, o que não impede que determinados trabalhos se encaixem em mais de uma categoria. O critério principal para o enquadramento dos trabalhos nas categorias foi a preponderância dos temas abordados pelos autores no contexto de cada pesquisa. Assim, tabela a seguir apresenta os trabalhos que se inserem na primeira categoria temática, agrupados por tipo de pesquisa, ordem cronológica de publicação e temática investigada: 
BARROS, L. C. Retrospectiva histórica ... preparação da performance musical. Per Musi, Belo Horizonte, n.31, 2015, p.284-299.

\begin{tabular}{|c|c|c|c|}
\hline $\begin{array}{l}\text { TIPO DE } \\
\text { PESQUISA }\end{array}$ & AUTOR (ES) & ANO & TEMÁTICA \\
\hline \multirow{9}{*}{$\begin{array}{l}\text { Pesquisas } \\
\text { experimentais } \\
\text { e pesquisas } \\
\text { descritivas } \\
\text { com } \\
\text { delineamento } \\
\text { experimental }\end{array}$} & GRUSON & 1988 & Análise da prática $x$ estratégias de estudo \\
\hline & COFFMAN & 1990 & $\begin{array}{l}\text { Tipos de prática x influência na otimização do } \\
\text { aprendizado }\end{array}$ \\
\hline & BARRY & 1992 & $\begin{array}{l}\text { Comportamento no estudo; efeitos da prática } \\
\text { estruturada/formal e da prática livre/informal }\end{array}$ \\
\hline & ERICSSON et al & 1993 & Prática deliberada \\
\hline & ERICSSON e KRAMPE & 1996 & Prática deliberada \\
\hline & DRAKE e PALMER & 2000 & Análise da prática, planejamento \\
\hline & ROSTRON e BOTTRILL & 2000 & $\begin{array}{l}\text { Estrutura da prática } x \text { efeitos no rendimento da } \\
\text { execucão }\end{array}$ \\
\hline & $\begin{array}{l}\text { WILLIAMON e } \\
\text { VALENTINE }\end{array}$ & 2000 & Relação quantidade x qualidade da prática \\
\hline & $\begin{array}{l}\text { WILLIAMON e } \\
\text { VALENTINE }\end{array}$ & 2002b & $\begin{array}{l}\text { A organização da prática a partir da estrutura formal } \\
\text { da peça }\end{array}$ \\
\hline \multirow{8}{*}{$\begin{array}{l}\text { Estudos de } \\
\text { caso }\end{array}$} & MIKLASZEWSKI & 1989 & Análise da preparação de um repertório \\
\hline & CHAFFIN e IMREH & $\begin{array}{l}1997 \\
2001 \\
2002\end{array}$ & $\begin{array}{l}\text { Análise da prática de uma pianista-concertista; } \\
\text { memorização }\end{array}$ \\
\hline & $\begin{array}{l}\text { CHAFFIN; IMREH e } \\
\text { CRAWFORD² }\end{array}$ & 2002 & $\begin{array}{l}\text { Acompanhamento do processo de aprendizagem de } \\
\text { uma obra de J.S.Bach por uma pianista-concertista }\end{array}$ \\
\hline & $\begin{array}{l}\text { RENWICK e } \\
\text { MCPHERSON }\end{array}$ & 2002 & $\begin{array}{l}\text { Relação entre repertório e motivação para a prática } \\
\text { (análise do comportamento de estudo) de uma } \\
\text { clarinetista }\end{array}$ \\
\hline & $\begin{array}{l}\text { CHAFFIN; IMREH; } \\
\text { LEMIEUX e CHEN }\end{array}$ & 2003 & $\begin{array}{l}\text { O papel da prática na solução de dificuldades } \\
\text { técnico-musicais em músicos proficientes }\end{array}$ \\
\hline & $\begin{array}{l}\text { CHAFFIN; LEMIEUX e } \\
\text { CHEN }\end{array}$ & 2004 & $\begin{array}{l}\text { Diferenças interpretativas em execuções de uma } \\
\text { mesma peça }\end{array}$ \\
\hline & $\begin{array}{l}\text { CHAFFIN; LISBOA e } \\
\text { LOGAN }\end{array}$ & 2005 & $\begin{array}{l}\text { Acompanhamento do processo de aprendizagem até } \\
\text { a performance }\end{array}$ \\
\hline & CHAFFIN e LOGAN & 2006 & $\begin{array}{l}\text { A preparação de uma concertista para uma } \\
\text { performance }\end{array}$ \\
\hline \multirow{7}{*}{$\begin{array}{l}\text { Estudos com } \\
\text { entrevista } \\
\text { e/ou } \\
\text { questionário }\end{array}$} & SOSNIAK & 1985 & $\begin{array}{l}\text { Estudo de entrevistas com } 21 \text { pianistas concertistas } \\
\text { sobre seu desenvolvimento profissional }\end{array}$ \\
\hline & SLOBODA e HOWE & $1991 \mathrm{a}$ & $\begin{array}{l}\text { Estudo de entrevistas com } 42 \text { jovens alunos sobre } \\
\text { influências na formação instrumental }\end{array}$ \\
\hline & HALLAM & $1995 a$ & $\begin{array}{l}\text { Estudo de entrevistas com } 22 \text { músicos sobre a } \\
\text { prática de estudo }\end{array}$ \\
\hline & HALLAM & 1995b & $\begin{array}{l}\text { Estudo de entrevistas com } 22 \text { músicos sobre a } \\
\text { relação entre aprendizagem e interpretação }\end{array}$ \\
\hline & HALLAM & $1997 b$ & $\begin{array}{l}\text { Estudo de entrevistas com } 22 \text { músicos profissionais e } \\
55 \text { iniciantes: análise da prática }\end{array}$ \\
\hline & DANIEL & 2001 & $\begin{array}{l}\text { Estudo com questionário para } 35 \text { instrumentistas } \\
\text { sobre a auto-avaliação da prática e da performance }\end{array}$ \\
\hline & BURLAND e DAVIDSON & 2002 & $\begin{array}{l}\text { Estudo de entrevistas com } 18 \text { músicos de nível de } \\
\text { excelência fatores atuantes na formação profissional }\end{array}$ \\
\hline $\begin{array}{l}\text { Levantamento } \\
\text { /Survey }\end{array}$ & SLOBODA e DAVIDSON & 1996 & $\begin{array}{l}\text { Levantamento com } 257 \text { crianças e jovens sobre seu } \\
\text { desenvolvimento musical }\end{array}$ \\
\hline
\end{tabular}


BARROS, L. C. Retrospectiva histórica ... preparação da performance musical. Per Musi, Belo Horizonte, n.31, 2015, p.284-299.

\begin{tabular}{|l|l|l|l|}
\hline \multirow{5}{*}{} & $\begin{array}{l}\text { SLOBODA; MOORE; } \\
\text { DAVIDSON; HOWE }\end{array}$ & 1996 & $\begin{array}{l}\text { Levantamento 257 crianças e jovens sobre a relação } \\
\text { prática e desenvolvimento da execução }\end{array}$ \\
\cline { 2 - 4 } & HARNISCHMACHER & 1997 & $\begin{array}{l}\text { Levantamento com 151 instrumentistas sobre o efeito } \\
\text { das diferenças individuais na estruturação da prática }\end{array}$ \\
\cline { 2 - 4 } & JORGENSEN & 1997 & $\begin{array}{l}\text { Levantamento com 182 alunos para verificar o tempo } \\
\text { despendido na prática }\end{array}$ \\
\cline { 2 - 4 } & KOTSKA & 2002 & $\begin{array}{l}\text { Levantamento com 261 alunos e professores sobre } \\
\text { atitudes e expectativas em relação à prática }\end{array}$ \\
\cline { 2 - 4 } & JORGENSEN & 2002 & $\begin{array}{l}\text { Levantamento com bacharelandos sobre a relação } \\
\text { entre nível de habilidade e quantidade de estudo }\end{array}$ \\
\cline { 2 - 4 } & $\begin{array}{l}\text { MCPHERSON e } \\
\text { MCCORMICK }\end{array}$ & $\begin{array}{l}\text { Levantamento com 332 instrumentistas sobre o papel } \\
\text { da auto-eficiência na preparação da performance } \\
\text { musical e nos provas de execução }\end{array}$ \\
\cline { 2 - 3 } & SMITH & $\begin{array}{l}\text { Levantamento com 344 instrumentistas sobre o } \\
\text { comportamento de estudo e análise de estratégias }\end{array}$ \\
\hline TIPO DE PESQUISA & QUANTIDADE DE PUBLICAÇÕES \\
\hline $\begin{array}{l}\text { Pesquisas experimentais e pesquisas descritivas } \\
\text { com delineamento experimental }\end{array}$ & 9 \\
\hline Estudos de caso & 10 \\
\hline Estudos com entrevista e/ou questionário & 7 \\
\hline Levantamentos / Survey & 8 \\
\hline $\begin{array}{l}\text { TOTAL DE PESQUISAS EMPIRICAS } \\
\text { NA TEMÁTICA 1 }\end{array}$ & 34 \\
\hline
\end{tabular}

Ex.1 - Tabela de temáticas relativas à análise do comportamento durante o estudo; organização, características e tipos de prática.

A próxima tabela identifica os trabalhos que se inserem na segunda categoria temática (temas que abordam as estratégias de estudo), agrupados por tipo de pesquisa, ordem cronológica de publicação e temática investigada:

\begin{tabular}{|c|c|c|c|}
\hline $\begin{array}{l}\text { TIPO DE } \\
\text { PESQUISA }\end{array}$ & AUTOR (ES) & ANO & TEMÁTICA \\
\hline \multirow{8}{*}{$\begin{array}{l}\text { Pesquisas } \\
\text { experimentais } \\
\text { e pesquisas } \\
\text { descritivas } \\
\text { com } \\
\text { delineamento } \\
\text { experimental }\end{array}$} & ROSENTHAL et al. & 1988 & $\begin{array}{l}\text { Efeitos de cinco estratégias de estudo sobre a } \\
\text { execução ao aprender uma nova peça musical }\end{array}$ \\
\hline & LIM e LIPPMAN & 1990 & Tipos de estudo mental no processo de memorização \\
\hline & $\begin{array}{l}\text { CAMP BINKOFSKI, } \\
\text { HALSBAND }\end{array}$ & 1994 & $\begin{array}{l}\text { O papel da percepção do agrupamento rítmico } \\
\text { (estratégia de agrupar notas para memorizar) na prática } \\
\text { como evidência para o desenvolvimento motor }\end{array}$ \\
\hline & PARNCUTT et al. & 1997 & $\begin{array}{l}\text { Estudo do dedilhado utilizado por } 28 \text { pianistas durante a } \\
\text { leitura de uma nova peça }\end{array}$ \\
\hline & $\begin{array}{l}\text { BURNSED e } \\
\text { HUMPHRIES }\end{array}$ & 1998 & $\begin{array}{l}\text { Pesquisa experimental com } 24 \text { sujeitos, examinando o } \\
\text { efeito da reverter a importância das mãos, ênfase na } \\
\text { m.e., no desenvolvimento da habilidade pianística }\end{array}$ \\
\hline & COSTA & 1999 & Aplicação de diferentes tipos de estratégias de estudo \\
\hline & HENLEY & 2001 & Estratégias sobre tempo/andamento \\
\hline & HALLAM & $2001 b^{3}$ & O desenvolvimento musical; estratégias de estudo \\
\hline \multirow[t]{2}{*}{$\begin{array}{l}\text { Estudos de } \\
\text { caso }^{4}\end{array}$} & NIELSEN & $\begin{array}{l}1997 \\
1999 a / b \\
2001\end{array}$ & $\begin{array}{l}\text { Auto monitoramento no aprendizado de estratégias } \\
\text { durante a prática }\end{array}$ \\
\hline & $\begin{array}{l}\text { PITTS; } \\
\text { DAVIDSON; } \\
\text { MCPHERSON }\end{array}$ & 2000 & $\begin{array}{l}\text { Tipos de estratégias cognitivas utilizadas por três } \\
\text { instrumentistas durante a prática }\end{array}$ \\
\hline Estudos com & HALLAM & $1997 a$ & Estudo com entrevista com 22 músicos profissionais e \\
\hline
\end{tabular}


BARROS, L. C. Retrospectiva histórica ... preparação da performance musical. Per Musi, Belo Horizonte, n.31, 2015, p.284-299.

\begin{tabular}{|l|l|l|l|}
\hline $\begin{array}{l}\text { entrevista } \\
\text { e/ou } \\
\text { questionário }\end{array}$ & $2001 \mathrm{a}$ & $\begin{array}{l}\text { 55 alunos iniciantes sobre o desenvolvimento de } \\
\text { estratégias metacognitivas }\end{array}$ \\
\hline $\begin{array}{l}\text { Levantamento } \\
\text { ISurvey }\end{array}$ & $\begin{array}{l}\text { BARRY e } \\
\text { MCARTHUR }\end{array}$ & 1994 & $\begin{array}{l}\text { Levantamento com 94 professores sobre o ensino de } \\
\text { estratégias de estudo }\end{array}$ \\
\cline { 2 - 3 } & NIELSEN & 2004 & $\begin{array}{l}\text { Levantamento 130 músicos avançados sobre utilização } \\
\text { de estratégias de estudo e o papel da auto-eficiência }\end{array}$ \\
\hline TIPO DE PESQUISA & QUANTIDADE DE PUBLICAÇÔES \\
\hline $\begin{array}{l}\text { Pesquisas experimentais e pesquisas descritivas } \\
\text { com delineamento experimental }\end{array}$ & 8 \\
\hline Estudos de caso & 5 \\
\hline Estudos com entrevista e/ou questionário & 1 \\
\hline Levantamentos / Survey & 2 \\
\hline $\begin{array}{l}\text { TOTAL DE PESQUISAS EMPÍRICAS } \\
\text { NA TEMÁTICA 2 }\end{array}$ & 16 \\
\hline
\end{tabular}

Ex.2 - Tabela de temáticas que abordam as estratégias de estudo.

A última tabela identifica os trabalhos que se inserem na terceira categoria temática (temáticas que abordam a representação mental da música e processos cognitivos envolvidos na memorização), agrupados por tipo de pesquisa, ordem cronológica de publicação e temática investigada:

\begin{tabular}{|l|l|l|l|}
\hline $\begin{array}{l}\text { TIPO DE } \\
\text { PESQUISA }\end{array}$ & AUTOR (ES) & ANO & TEMÁTICA \\
\hline $\begin{array}{l}\text { Pesquisas } \\
\text { experimentais e } \\
\text { pesquisas } \\
\text { descritivas com } \\
\text { delineamento } \\
\text { experimental }\end{array}$ & LEHMANN & 1997 & Representação mental da música \\
\cline { 2 - 4 } & WILLIAMON & 1999 & $\begin{array}{l}\text { Os efeitos da performance memorizada sobre o } \\
\text { ouvinte }\end{array}$ \\
\cline { 2 - 4 } & MISHRA & 2002 & $\begin{array}{l}\text { Análise de estratégias eficiente e ineficientes na } \\
\text { memorização }\end{array}$ \\
\cline { 2 - 4 } & $\begin{array}{l}\text { WILLIAMON; } \\
\text { VALENTINE }\end{array}$ & $2002 \mathrm{a}$ & O papel da estrutura formal da peça na memorização \\
\hline $\begin{array}{l}\text { Estudos com } \\
\text { entrevista } \\
\text { e/ou } \\
\text { questionário; } \\
\text { levantamento }\end{array}$ & HALLAN & $1997 a$ & $\begin{array}{l}\text { Entrevista com 22 músicos profissionais e 55 } \\
\text { iniciantes sobre o desenvolvimento de estratégias de } \\
\text { memorização }\end{array}$ \\
\cline { 2 - 4 } & HOLMES & 2005 & $\begin{array}{l}\text { Estudo de entrevista com dois músicos profissionais } \\
\text { sobre os aspectos cognitivos, representação mental e } \\
\text { memorização }\end{array}$ \\
\hline \multicolumn{2}{|l|}{ TIPO DE PESQUISA } & QUANTIDADE DE PUBLICAÇÕES \\
\hline $\begin{array}{l}\text { Pesquisas experimentais e pesquisas descritivas } \\
\text { com delineamento experimental }\end{array}$ & 4 \\
\hline \begin{tabular}{l} 
Estudos com entrevista e/ou questionário \\
\hline
\end{tabular} & 2 \\
\hline $\begin{array}{l}\text { TOTAL DE PESQUISAS EMPÍRICAS } \\
\text { NA TEMÁTICA 3 }\end{array}$ & 6 \\
\hline
\end{tabular}

Ex.3 - Tabela de temáticas que abordam a representação mental da música e os processos cognitivos envolvidos na memorização.

As fontes bibliográficas sobre o planejamento da execução mostram que os trabalhos sobre a análise do comportamento durante o estudo, sua organização, características e tipos de prática constituem o foco temático principal dessa linha de 
pesquisa, totalizando trinta e quatro trabalhos. Alguns assuntos, inseridos nas categorias anteriormente citadas, são apoiados em pesquisas desenvolvidas principalmente nos campos da psicologia da música (sob tendências da psicologia cognitiva) e da neurociência. Assim, alguns termos e teorias de outras áreas do conhecimento foram aplicados e transformados de acordo com as características e os objetivos da área de Práticas Interpretativas. O termo prática deliberada de estudo foi criado justamente para ilustrar essa interferência interdisciplinar na linha de pesquisa do planejamento da execução para designar as estratégias de estudo que levam o pianista a alcançar um alto nível na execução instrumental. Segundo GABRIELSSON, o termo significa uma "série de atividades cuidadosamente estruturadas objetivando a otimização da execução e que pressupõe um elevado nível de motivação e de esforço estendido" (2003, p.241). Desse modo, o resultado final da execução depende do trabalho precedente e inclui a análise e a verificação dos processos e estratégias de sua preparação. Esta denominação é empregada em trabalhos de ERICSSON, KRAMPE e TESCH-ROMER (1993); ERICSSON e CHARNESS (1994); ERICSSON e KRAMPE (1996); ERICSSON e NEIL (1994); LEHMANN et al. (1996, 1997, 1998) e SLOBODA et al. (1996), os quais postulam que o principal fator para o desempenho instrumental de excelência é dado pela quantidade acumulada de horas de estudo - um mínimo de dez anos na prática deliberada, eficazmente distribuída (GABRIELSSON, 2003, p.241).

Grande parte dos estudos sobre a temática "prática deliberada" em música foi realizada através de pesquisas descritivas com delineamento experimental. $O$ trabalho pioneiro sobre esse tema é a pesquisa experimental de ERICSSON et al., (1993). Esse estudo resultou em um dos referenciais mais relevantes em relação à prática deliberada de estudo, sendo citado em muitos artigos sobre o planejamento da execução instrumental. Com isso, através de entrevistas, uso de diários de acompanhamento da prática, testes experimentais, observações e acompanhamento em dois estudos descritivos, um com violinistas e outro com pianistas, chegou-se à conclusão de que muitas características atribuídas ao talento inato provêm, na realidade, de uma extensa e planejada prática de estudo ocorridas em um período mínimo de dez anos. A prática deliberada também assegura que o estudo diário do instrumento engloba, além de suas etapas e atividades estruturadas, outros aspectos subjacentes como fatores psicológicos, cognitivos, físicos, emocionais, dentre outros. As pesquisas sobre a prática deliberada são influenciadas pelos procedimentos metodológicos utilizados pela área da psicologia, tendendo a realizar estudos de caráter prático e empírico.

Certas correntes de estudos dentro do planejamento da execução contrariam alguns resultados apregoados pelos estudiosos da prática deliberada, a exemplo de WILLIAMON e VALENTINE (2000 e 2002). Esses pesquisadores acompanharam o aprendizado e memorização de obras de J. S. Bach com vinte e dois pianistas, divididos em quatro níveis de habilidade. O trabalho opõe-se ao argumento de que a quantidade de horas de estudo é fator determinante para a qualidade da execução. Ao contrário, notou-se que o conteúdo e a qualidade individual da prática de estudo precisam ser investigados como fatores determinantes da habilidade musical, sugerindo que não se considere somente o enfoque quantitativo ao examinar a 
constituição da habilidade musical, mas, sobretudo, os aspectos qualitativos e de conteúdo dessa prática. Desse modo, não é somente a quantidade de horas de estudo que determina o resultado, mas principalmente como o tempo consumido nessa prática é distribuído e organizado e se é capaz de produzir resultados altamente eficazes. Considerando as implicações da relação entre quantidade e qualidade da prática como potencial temático para pesquisas, WILLIAMON ressalta que, ainda, não foi claramente determinado até que ponto a extensão e quantidade da prática resultam em qualidade (2000, p.3). HALLAM (2001b, p.7) aponta que nem todas as evidências corroboram o argumento sobre o número de horas como fator determinante para o nível de habilidade, visto que outros aspectos como o conhecimento musical prévio e a qualidade da prática podem atuar como fatores interferentes no desempenho musical.

As pesquisas sobre o comportamento de estudo podem elucidar muitas indagações referentes à aquisição do conteúdo musical. Dentre os resultados encontrados, a partir da observação de músicos com diferentes níveis de desempenho, notou-se que a condução da prática depende do grau de habilidade do músico. Verificou-se que instrumentistas profissionais demonstram extensivas habilidades metacognitivas e conseguem avaliar seus pontos fracos e fortes, ou seja, aprendem como estudar e desenvolvem apropriadas estratégias para a prática (CHAFFIN et al., 2002; HALLAM, 2001; WILLIAMON, 2004; dentre outros). MCPHERSON e MCCORMICK (2003) indicaram que os alunos que obtiveram resultados de execução superiores foram os que exibiram maiores níveis de estratégias cognitivas, tais como estudar a música mentalmente, avaliar criticamente seus esforços e organizar sua prática de estudo para alcançar resultados produtivos. Isto mostra que a utilização de aspectos cognitivos durante o estudo é uma tática importante para refinar o trabalho musical.

O segundo foco temático em número de publicações são as pesquisas que examinaram as estratégias de estudo, totalizando dezesseis trabalhos. A temática, considerando sua especificidade e aplicação prática, é a que possui maior potencial investigativo para ser examinada por meio de pesquisas empíricas - muitas estratégias nunca foram testadas cientificamente, mesmo sendo embasadas em um contexto teórico ou informalmente testadas dentro da experiência do ensino instrumental. Em relação ao número de pesquisas empíricas sobre o planejamento, existem poucos trabalhos com delineamento experimental sobre as estratégias de estudo empregadas na prática instrumental. Por outro lado, existem inúmeras referências puramente teóricas que abordam e sugerem técnicas e estratégias a serem aplicadas no aprendizado do repertório. Esse conhecimento pode ser fonte para procedimentos que possam testar as teorias formuladas.

Diversos trabalhos (HALLAM, 1995, 2001; BARRY, 1992; COSTA, 1999; WILLIAMON, 2004; ERICSSON et al., 1993, dentre outros) mostraram que o uso flexível de estratégias de estudo é uma das características da prática de músicos em nível de expertise. Isto foi verificado na pesquisa participante de CHAFFIN et al., (2002), em que a pianista valeu-se de um grande número de estratégias e utilizavaas de acordo com cada situação encontrada durante a prática. Existem, assim, incontáveis possibilidades investigativas a serem examinadas dentro dessa temática 
e que poderiam indicar maneiras de aperfeiçoar a prática instrumental por meio da validação dessas estratégias. Isto poderia revelar quais seriam as estratégias utilizadas por músicos profissionais, em que contexto e como são aplicadas e qual o resultado alcançado.

Embora em menor número (seis, no total), provavelmente as temáticas envolvendo os processos de memorização e de representação mental da música ${ }^{5}$ são, dentro do planejamento da execução instrumental, as que apresentam um dos maiores níveis de desenvolvimento investigativo, englobando trabalhos de resultados substanciais e de reconhecido mérito. Embora não tenha inserido os trabalhos de CHAFFIN et al. (1997, 2002, 2006) na tabela Ex.3, o incluo nesta análise, pois seus trabalhos examinam a memória proficiente em músicos, mesmo que não especificamente a representação mental. A consistência científica dos trabalhos dessa temática devese à influência do embasamento teórico das pesquisas realizadas no campo da psicologia e da neurociência e de pesquisadores dessas áreas, os quais atuam em investigações na área de Práticas Interpretativas, como CHAFFIN (2002); DAVIDSON (2004); ERICSSON et al., (1993); GINSBORG (CHAFFIN et al., 2006b); JORGENSEN (2000); JUSLIN (2002); LEHMANN (1997); PALMER (1997); WILLIAMON e VALENTINE (2000), dentre outros. Segundo HIGUCHI:

As pesquisas neurocientíficas proporcionaram informações a respeito do processo de memorização que permitiram elaborar procedimentos eficientes para desenvolver uma memorização mais adequada para um aprendizado pianístico elaborado (HIGUCHI, 2005, p.222).

Os estudos envolvendo a temática da memorização variam quanto ao método de pesquisa. Um dos livros de maior relevância e citação em artigos sobre a temática da memorização é o estudo de caso realizado por CHAFFIN et al., (2002). Esse trabalho apresentou o sistemático processo de observação de uma pianista concertista preparando o $3^{\circ}$ Movimento do Concerto Italiano de J. S. Bach (desde a leitura à gravação de um CD dessa obra), apresentando uma rigorosa análise de sua prática. Outras pesquisas examinaram a temática a partir de um foco menos abrangente, como o de MISHRA (2002), utilizando apenas um pequeno trecho de uma peça musical para verificar o tipo de estratégia de memorização empregada pelo músico e seguindo modelos investigativos semelhantes aos estudos pioneiros realizados por RUBIN-RABSON (1937, 1939, 1940, 1941). Embora haja avanço nas temáticas sobre a memorização, ainda há muito o que se investigar, principalmente se, além de descrever as estratégias de memorização que os músicos utilizam, essas forem selecionadas pelo pesquisador e aplicadas com sujeitos, a fim de comparar as estratégias que melhor possibilitam a retenção do conteúdo musical na memória de longa duração.

\section{5 - Conclusão}

A análise crítica e a reflexão sobre as fontes bibliográficas do presente artigo mostraram que o planejamento da execução instrumental é uma das linhas de 
pesquisa mais relevantes e promissoras das Práticas Interpretativas, justamente por apresentar abundante fonte de material temático a ser examinado por pesquisas empíricas. A retrospectiva histórica aponta que essa vertente investigativa é relativamente nova e possui poucos trabalhos empíricos se comparada às demais áreas do conhecimento. As três categorias temáticas propõem sistematizar 0 direcionamento investigativo dos trabalhos, indicando os tipos de pesquisa utilizados, as temáticas mais examinadas e os principais autores. Contudo, existem inúmeras possibilidades de validação científica e experimentação prática dos conceitos, princípios e teorias formuladas que têm sua origem nos trabalhos puramente teóricos ou na prática vivenciada de especialistas da área instrumental (a tradicional transmissão oral do conhecimento) que podem ser examinadas por meio de diferentes técnicas de pesquisa. Depreende-se, então, que o planejamento da execução instrumental tem a maior quantidade de problemas de pesquisa inexplorados, pois a linha de investigação compreende todo o processo de aprendizagem de um determinado repertório musical: inicia-se no primeiro contato com a partitura (leitura à primeira vista) até o resultado final da performance (apresentação da obra). Isso também corrobora para que a mesma seja estabelecida como uma das linhas que mais se vincula ao campo da didática do instrumento, transformando-se numa justificativa para incentivar estudos empíricos que possam descrever e validar, ou não, aspectos fundamentais da prática instrumental.

\section{Referências}

BARRY, N. The effects of practice strategies, individual differences in cognitive style, and gender upon technical accuracy and musicality of student instrumental performance. Psychology of Music, v. 20 n.2, p.112-123, 1992.

BORÉM, F.; BENDA, R.; LAGE, G.; MORAES, L. Aprendizagem motora na performace musical: reflexões sobre conceitos e aplicabilidade. PER MUSI, v. 5 e 6, p.14-37, 2002.

BURNSED, V.; HUMPHRIES, S. The effects of reversing the roles of the hands on the development of piano performance skill: a preliminary investigation. Psychology of Music, v.26, p.89-96, 1998.

CHAFIN, R.; IMREH, G.; CRAWFORD, M. Practicing perfection: memory and piano performance. Mahwah. NJ: Erlbaum, 2002.

CHAFFIN, R.; LOGAN, C..; LISBOA, T. An inquiry into the dynamics of performance investigating conception and attention from practice to performance on the cello. In: Simpósio Internacional de Cognição e Artes Musicais, $1^{\circ}$., 2005, Curitiba. Anais. Curitiba: Deartes - UFPR, 2005. p. 228-236.

CLARKE, E. F. Generative principles in music performance. In: SLOBODA (ed) Generative processes in music: the psychology of performance, improvisation and composition. Oxford: Clarendon Press, 1988, p.1-26.

COFFMAN, Don. Effects of mental practice, physical practice and knowledge of results on piano performance. British Journal of Music Education, v. 38 n.3, p.187-196, 1990.

COSTA, Doris. An investigation into instrumental pupils' attitudes to varied, structured practice: two methods of approach. British Journal of Music Education, v. 16 n.1, p.65-77, 1999. 
BARROS, L. C. Retrospectiva histórica ... preparação da performance musical. Per Musi, Belo Horizonte, n.31, 2015, p.284-299.

DAVIDSON, J. The Music Practitioner: Research for the Music Performer, Teacher, and Listener. Burlington: Ashgate Publishing, 2004.

DRAKE, C.; PALMER, C. Skill acquisition in music performance: relations between planning and temporal control. Cognition, 74, p.1-32, 2000.

ERICSSON, K. Anders; KRAMPE, Ralf; TESCH-ROMER, Clemens. The role of deliberate practice in the acquisition of expert performance. Psychological Review, v. 100 n.3, p.363-406, 1993.

ERICSSON, K. Anders; KRAMPE, Ralf. Maintaining excellence: deliberate practice and elite performance in young and older pianists. Journal of Experimental Psychology: General, v.125 n.4, p.331-359, 1996.

ERICSSON, K. CHARNESS, N. Expert performance: its structure and acquisition. American Psychologist, v. 49, p.725-747, 1994.

GABRIELSSON, A. Music performance research at the millennium. Psychology of Music, v. 31, p.221$272,2003$.

GRUSON, Linda. Rehearsal skill and musical competence: does practice makes perfect? In: SLOBODA, John. Generative Processes in Music: The Psychology of Performance, improvisation and Composition. Oxford: Clarendon Press, 1988, p. 91-112.

HALLAM, S. The development of metacognition in musicians: implications for education. British Journal of Music Education, v.18 n.1, p.27-39, 2001a.

. The development of expertise in young musicians: strategy use, knowledge acquisition and individual diversity. Music Education Research, v. 3 n.1, p. 7-23, 2001b.

. Professional musicians' orientations to practice: implications for teaching. British Journal

of Music Education, v. 12, p.3-19, 1995a.

The development of memorization strategies in musicians: implications for education.

British Journal of Music Education, v.14 n.1, p.87-97, 1997a.

HIGUCHI, Márcia. Memorização no aprendizado pianístico: A contribuição da neurociência. In:

Simpósio Internacional de Cognição e Artes Musicais, 1‥, 2005, Curitiba. Anais. Curitiba: Deartes UFPR, 2005. p. 221-227.

JORGENSEN, Harald. Student learning in higher instrumental education: who is responsible? British Journal of Music Education, v. 17 n.1, p.67-77, 2000.

JUSLIN; Patrik; PERSSON, Roland. Emotional Communication. In: PARNCUTT, R.; MCPHERSON, G. Science and Psychology of Music Performance. Oxford: Oxford University Press, 2002, p.219-235.

KRAMPE, R.T. Age-related changes in practice activities and their relation to musical performance skills. In: JORGENSEN, H.; LEHMANN, A. Does practice make perfect? Current theory and research on instrumental music performance. Oslo: Norwegian State Academy of Music, 1997, p. 165-78.

LEHMANN, A. C. Acquired mental representations in music performance: anecdotal and preliminary empirical evidence. In: JORGENSEN, H.; LEHMANN, A. Does practice make perfect? Current theory and research on instrumental music performance. Oslo: Norwegian State Academy of Music, 1997, p.141-63.

MCPHERSON, G.; MCCORMICK, J. The role of self-efficacy in a musical performance examination: an exploratory structural equation analysis. Psychology of Music, v. 31 n.1, p.37-51, 2003.

MANTURZEWSKA, Maria. A biographical study of the life-span development of professional musicians. Psychology of Music, v.18, p.112-139, 1990. 
BARROS, L. C. Retrospectiva histórica ... preparação da performance musical. Per Musi, Belo Horizonte, n.31, 2015, p.284-299.

MIKLASZEWSKI, K. A case study of a pianist preparing a musical performance. Psychology of Music, v. 17, p.95-109, 1989.

MISHRA, Jennifer. A qualitative analysis of strategies employed in efficient and inefficient memorization. Bulletin of the Council for Research in Music Education, n.152, p.74-86, 2002.

NIELSEN. Self-regulating learning strategies in instrumental music practice. Music Education Research, v.3 n.2, p.155-67, 2001.

O'NEILL, S.A. The role of practice in children's early musical performance achievement. In: JORGENSEN, H.; LEHMANN, A. Does practice make perfect? Current theory and research on instrumental music performance. Oslo: Norwegian State Academy of Music, 1997, p.53-70.

PALMER, C. Music performance. Annu. Rev. Psychol, v.48, p.115-138, 1997.

REID. Preparing for performance. In: RINK, J. Musical performance: a guide to understanding. Cambridge: Cambridge University Press, 2002, p.102-112.

ROSENTHAL; WILSON; EVANS; GREENWALT. Effects of different practice conditions on advanced instrumentalists' performance accuracy. Journal of Research in Music Education, v. 36, p.250-257, 1988.

ROSTRON, A.; BOTTRILL, S. Are pianists different? Some evidence from performers and nonperformers. Psychology of Music, v.28, p.43-61, 2000.

SLOBODA, J.A.; DAVIDSON, J.W.; HOWE, M.J.A.; MOORE, D.G. The role of practice in the development of performing musicians. British Journal of Psychology, v. 87, p.287-309, 1996.

WILLIAMON, Aaron. Musical excellence: strategies and techniques to enhance performance. Oxford: Oxford University Press, 2004.

WILLIAMON, A.; THOMPSON, S. Evaluating evaluation: musical performance assessment as a research tool. Music Perception, v. 21 n.1, p.21-41, 2003.

Psychology and the music practitioner. In: DAVIDSON, J. The Music Practitioner: Research for the Music Performer, Teacher, and Listener. Ashgate Publishing, 2004, p.9-26.

WILLIAMON, A.; VALENTINE, E. Quantity and quality of musical practice as predictors of performance quality. The British Journal of Psychology, 91, p. 353-376, Ago. 2000.

2002a. . The role of retrieval structures in memorizing music. Cognitive Psychology, n.44, p.1-32,

WOODY, Robert. Explaining expressive performance: component cognitive skills in an aural modeling task. Journal of Research in Music Education, v. 51 n.1, p.51-63, 2003.

\section{Notas}

1 Um dos referenciais para o presente artigo foi o trabalho de GABRIELSSON, A. (2003), publicado no periódico Psychology of Music e tendo como título "Music performance research at the milennium". Gabrielsson fez um mapeamento sucinto, revisão e análise da estrutura organizacional de dez linhas de pesquisa em Práticas Interpretativas, a partir de mais de quinhentos artigos compilados em revistas e periódicos entre 1995 e 2002. A opção por esse referencial se fundamenta na evidência de que Gabrielsson foi o primeiro pesquisador a indicar esse foco investigativo (linha de pesquisa n.5), além de descrever de forma concisa e conceitual o panorama das linhas de pesquisa em Práticas Interpretativas, indicando as principais fontes e fornecendo um breve resultado de algumas dessas 
investigações. As linhas foram estabelecidas por ordem decrescente de número de trabalhos, a saber: 1. Medição da Execução Instrumental (Measurements of performance); 2. Fatores Psicológicos e Sociais da Execução Instrumental (Psychological and social factors); 3. Fatores Físicos na Execução Instrumental (Physical factors in performance); 4. Processos Motores na Execução Instrumental (Motor processes in performance); 5. Planejamento da Execução Instrumental (Performance planning); 6. Modelos da Execução Instrumental (Models of music performance); 7. Leitura à Primeira Vista (Sight reading); 8. Avaliação da Execução Instrumental (Performance evaluation); 9. Improvisação (Improvisation) e; 10. Feedback na Execução Instrumental (Feedback in performance).

${ }^{2}$ Os estudos de caso de CHAFFIN et al., devido a sua abrangência e detalhamento, também, se inserem nas outras tabelas temáticas.

${ }^{3}$ Embora essa pesquisa faça parte do mesmo estudo com entrevista, relatado nos demais artigos de Hallam, eu o considerei como uma pesquisa descritiva com delineamento experimental, visto que esse artigo abordou, principalmente, o resultado das gravações da prática de uma nova peça musical realizadas por 55 instrumentistas.

${ }^{4}$ Alguns autores como BOGDAN e BIKLEN (1994) adotam o termo estudo multicasos ou estudos de casos múltiplos para designar estudos de caso que lidam dois ou mais sujeitos. O termo em inglês é multiple case studies. Selecionei alguns estudos de caso que tiveram mais de dois participantes, como os de NIELSEN (1999, 2001) com dois organistas; e PITTS el al. (2000) com três instrumentistas. Para o presente trabalho decidi manter a mesma terminologia que esses autores empregaram em suas pesquisas: estudos de caso.

5 Termo utilizado pelos pesquisadores da psicologia da música para designar a apreensão, compreensão e concepção imaginária de uma peça musical, a qual é fundamentada nas experiências musicais prévias do músico. Entre os trabalhos mais relevantes sobre a representação mental estão: LEHMANN (1997), ERICSSON (1997), LEHMANN et al. (2007); LEHMANN e DAVIDSON (2002). Segundo Lehmann, o conceito de representação mental é ambíguo na psicologia, referindo-se à reconstrução interna de um mundo exterior. Nas palavras de um renomado pianista e professor, como NEUHAUS (1967), esse conceito poderia significar "a imagem artística" da obra musical (LEHMANN et al. 2007, p.19).

Luís Cláudio Barros é professor de piano do Departamento de Música da Universidade do Estado de Santa Catarina (UDESC). É bacharel em piano pela Faculdade de Música do Espírito Santo, sob orientação da pianista Célia Ottoni, mestre em piano pela Eastman School of Music (NY/EUA), sob orientação da Dra. Nelita True e doutor em música na área de Práticas Interpretativas pela UFRGS, tendo orientação acadêmica com a Dra. Any Raquel Carvalho e artística com os doutores Ney Fialkow e Catarina Domenici. Entre 2006 e 2007 realizou o Estágio de Doutorado na Universidade de Connecticut (EUA) sob a co-orientação do Dr. Roger Chaffin, tendo trabalhado no Laboratório de Performance e Psicologia da Música. É detentor de seis primeiros lugares em Concursos Nacionais de Piano. Tem desenvolvido atividades de pesquisa com as temáticas "planejamento da execução instrumental" e didática do instrumento. Fez parte do "Piano Faculty" no 14th World Piano Pedagogy Conference, nos EUA, além de realizar recitais solo e de câmara no Brasil e no exterior. 